Check for updates

Cite this: Chem. Commun., 2020, 56,6989

Received 13th January 2020,

Accepted 12th May 2020

DOI: $10.1039 / \mathrm{d} 0 \mathrm{cc} 00329 \mathrm{~h}$

rsc.li/chemcomm

\section{Silica-coated phosphorescent nanoprobes for selective cell targeting and dynamic bioimaging of pathogen-host cell interactions $\dagger$}

\author{
Federico lovino, (D) ab Padryk Merkl, ${ }^{a}$ Anastasia Spyrogianni, $\ddagger^{c}$ \\ Birgitta Henriques-Normark ${ }^{\mathrm{ab}}$ and Georgios A. Sotiriou (D) *a
}

\begin{abstract}
Fluorescence in vitro bioimaging suffers from photobleaching of organic dyes, thus, functional probes with superior photostability are urgently needed. Here, we address this challenge by developing novel silica-coated nanophosphors that may serve as superior luminescent nanoprobes compatible with conventional fluorescence microscopes. We specifically explore their suitability for dynamic in vitro bioimaging of interactions between bacterial pathogens and host cells, and further demonstrate the facile surface functionalization of the amorphous silica layer with antibodies for selective cell targeting.
\end{abstract}

Optical fluorescence microscopy is one of the most commonly employed tools when studying nanoparticle-cell interactions in vitro. In these studies nanoparticles are often labelled with an organic fluorophore (dye) that upon excitation at a specific wavelength emits light at a different wavelength, that is subsequently detected using the appropriate excitation and emission filters. A plethora of commercially-available organic dyes exist that offer a bright signal and are traditionally used for labelling biomolecules (e.g. proteins, peptides). However, a major drawback of organic dyes for bioimaging is their poor photostability. ${ }^{1}$ Even though useful images of nanoparticle-cell interactions may be obtained with these dyes at one time-point, it is not possible to perform dynamic imaging over time because such organic fluorophores rapidly degrade during continuous irradiation, thus losing their intensity and exhibiting the so-called photobleaching. ${ }^{2,3}$ Dynamic bioimaging enables tracking of interactions in real-time, obtaining not only spatial but also

\footnotetext{
${ }^{a}$ Department of Microbiology, Tumor and Cell Biology, Karolinska Institutet, SE-17165 Stockholm, Sweden. E-mail: georgios.sotiriou@ki.se

${ }^{b}$ Department of Clinical Microbiology, Karolinska University Hospital, SE-17176 Stockholm, Sweden

' Particle Technology Laboratory, Institute of Process Engineering, Department of Mechanical and Process Engineering, ETH Zurich, CH-8092 Zurich, Switzerland $\dagger$ Electronic supplementary information (ESI) available. See DOI: 10.1039/ d0cc00329h

\# Current address: Drug Formulation and Delivery, Institute of Pharmaceutical Sciences, Department of Chemistry and Applied Biosciences, ETH Zurich, CH-8093 Zurich, Switzerland.
}

temporal information of specific important events during an experiment, crucial in several fields including host-pathogen interaction studies.

One novel class of nanoparticles inherently luminescent and characterized by superior optical stability are nanophosphors. ${ }^{4-6}$ Nanophosphors are nanoparticles that typically consist of a crystal host matrix that is doped with rare earth ions and are routinely used in lighting. ${ }^{7}$ The excitation and emission of nanophosphors depends on both the crystalline material as well as the selection of the rare earth ions and in fact, by co-doping such nanoparticles with multiple rare earth ions it is possible to obtain color tunability. ${ }^{8}$ These materials may exhibit superior performance in biosensing in vitro, ${ }^{9,10}$ however, one major limitation of such nanophosphors lies at their excitation wavelength. The excitation spectra of most nanophosphors have strong and broad charge transfer (CT) bands in the UV-C $(\lambda=100-280 \mathrm{~nm})$ and UV-B $(\lambda=280-315 \mathrm{~nm})$ regions, ${ }^{11}$ in which, however, the standard optical components of conventional fluorescence microscopes strongly absorb and the shortest wavelength in which they operate is in the near UV region at $\lambda=315-400 \mathrm{~nm}$ (UV-A). These technical issues inhibit in vitro imaging with nanophosphors and conventional microscopes requiring complex and expensive solutions (e.g. quartz optical components or lasers as excitation). ${ }^{12,13}$

One strategy to tune the excitation bands of nanophosphors to UV-A is by co-doping $\mathrm{YVO}_{4}: \mathrm{Eu}^{3+}$ nanoparticles with $\mathrm{Bi}^{3+}$ ions. ${ }^{14-17}$ The $\mathrm{Bi}^{3+}$ doping shifts the excitation spectrum of $\mathrm{YVO}_{4}: \mathrm{Eu}^{3+}$ nanophosphors to near-UV spectral region rendering these nanoparticles suitable nanoprobes for in vitro bioimaging with conventional fluorescence microscopes ${ }^{18}$ and even exhibit attractive properties as imaging nanoprobes for correlative cathodoluminescence electron microscopy. ${ }^{19}$

Here, we develop novel bioimaging nanoprobes based on $\mathrm{YVO}_{4}: \mathrm{Eu}^{3+}, \mathrm{Bi}^{3+}$ nanoparticles and further advance the state-ofthe-art by in situ coating them during their gas-phase synthesis in a single step with a nanothin $(\sim 3 \mathrm{~nm})$ layer of amorphous silica $\left(\mathrm{SiO}_{2}\right) \cdot{ }^{20,21}$ We explore the optical stability of these nanoprobes and directly compare them with an organic dye by 
performing in vitro dynamic bioimaging of lung epithelial cells challenged with the bacterium Streptococcus pneumoniae. We demonstrate the facile biofunctionalization of these nanoprobes with antiCK8 antibody that readily binds to the type II cytoskeletal 8 keratin protein expressed in these lung epithelial cells. ${ }^{22}$ We perform cell experiments with conventional upright cell culture orientation (cells are placed on the bottom of wells), but also with inverted cell culture orientation ${ }^{23}$ in which the cells are placed on top of the well. Monitoring the luminescence from nanoprobes enables direct quantitative comparison of the targeting capacity of antiCK8-functionalized nanoprobes with non-functionalized ones, and functionalized with, bovine serum albumin (BSA).

An important characteristic of luminescent nanoprobes is to have a biocompatible surface that may be easily functionalized with biomolecules. One of the most understood such surface for bioconjugation is amorphous $\mathrm{SiO}_{2}$. The enclosed flame aerosol reactor employed in this study allows for the single-step production of coreshell nanoparticles that are encapsulated by a nanothin layer of $\mathrm{SiO}_{2}$ (Fig. 1a). In fact this scalable and reproducible process ${ }^{24}$ has been successfully employed to encapsulate a variety of materials such as $\mathrm{TiO}_{2},{ }^{20} \mathrm{Fe}_{2} \mathrm{O}_{3},{ }^{25} \mathrm{Ag},{ }^{26} \mathrm{Au},{ }^{27} \mathrm{Y}_{2} \mathrm{O}_{3}: \mathrm{Tb}^{3+},{ }^{28}$ to name a few. Fig. $1 \mathrm{~b}$ and $\mathrm{c}$ shows high resolution transmission electron microscope images of the $\mathrm{SiO}_{2}$-coated $\mathrm{YVO}_{4}: \mathrm{Eu}^{3+}, \mathrm{Bi}^{3+}\left(23 \mathrm{wt} \% \mathrm{SiO}_{2}\right)$ nanoparticles produced here in which the darker crystalline core is encapsulated by a smooth, nanothin $(\sim 3 \mathrm{~nm})$ amorphous $\mathrm{SiO}_{2}$ coating. The presence of $\mathrm{SiO}_{2}$ as a coating is further validated in Fig. 1d and e that shows high-angle annular dark field (HAADF) electron microscopy images and the corresponding elemental mapping (obtained by energydispersive X-ray spectroscopy-EDX, further images shown in ESI, $\dagger$ Fig. S1a) in which the core material (red dots, monitoring V) is easily distinguished from the shell (green dots, monitoring $\mathrm{Si}$ ).
The elemental composition is further validated by the EDX spectra of selected areas in these images (ESI, $\dagger$ Fig. S1b).

The crystallinity of the core material is confirmed by X-ray powder diffraction $(\mathrm{XRD})(\mathrm{ESI}, \dagger$ Fig. S2) that reveals the characteristic tetragonal $\mathrm{YVO}_{4}$ crystal phase with an average crystal diameter $d_{\mathrm{XRD}}=26 \mathrm{~nm}$ (calculated from the main diffraction peak at $24.9^{\circ}$ ). No Eu-oxide nor Bi-oxide peaks (with a minor $\mathrm{Y}_{2} \mathrm{O}_{3}$ phase of $3 \mathrm{wt} \%$ calculated by Rietveld refinement) are detected in the XRD pattern further indicating incorporation of both the $\mathrm{Eu}^{3+}$ and $\mathrm{Bi}^{3+}$ ions in the crystal host lattice. ${ }^{18}$ The incorporation of both $\mathrm{Eu}^{3+}$ and $\mathrm{Bi}^{3+}$ ions renders these nanoparticles luminescent by near-UV excitation with emission in the red part of the spectrum as shown in Fig. 1f, and these excitation and emission profiles allow them to be utilized with traditional fluorescence microscopes for live cell imaging studies with existing filter sets (e.g. excitation $350 \pm 50 \mathrm{~nm}$ (DAPI) and emission $620 \pm 26 \mathrm{~nm}$ (TRITC)). ${ }^{18}$

The presence of the amorphous $\mathrm{SiO}_{2}$ surface is further validated by the attenuated total reflection Fourier transform infrared spectra (FTIR-ATR) of the nanoprobes. Fig. $1 \mathrm{~g}$ shows such FTIR-ATR spectra in which the $\mathrm{Si}-\mathrm{O}-\mathrm{Si}$ and surface $-\mathrm{OH}$ bands and/or absorbed water around $1000 \mathrm{~cm}^{-1}$ and $3200 \mathrm{~cm}^{-1}$ are seen respectively. ${ }^{29}$ The hydrophilic amorphous $\mathrm{SiO}_{2}$ coating facilitates apart from nanoparticle dispersion in aqueous solutions (ESI, $\dagger$ Fig. S3), ${ }^{21}$ also biomolecule functionalization by physical adsorption, an increasingly popular route for decorating the surface of nanoparticles with targeting antibodies. ${ }^{30,31}$ Upon incubation of these nanoprobes with bovine serum albumin (BSA, mass ratio is 1:1) in phosphate buffered saline (PBS) at room temperature for $30 \mathrm{~min}$ followed by washing, the characteristic amide I and II bands from $\mathrm{BSA}^{32}$ appear around $1600 \mathrm{~cm}^{-1}$ indicating its presence on the
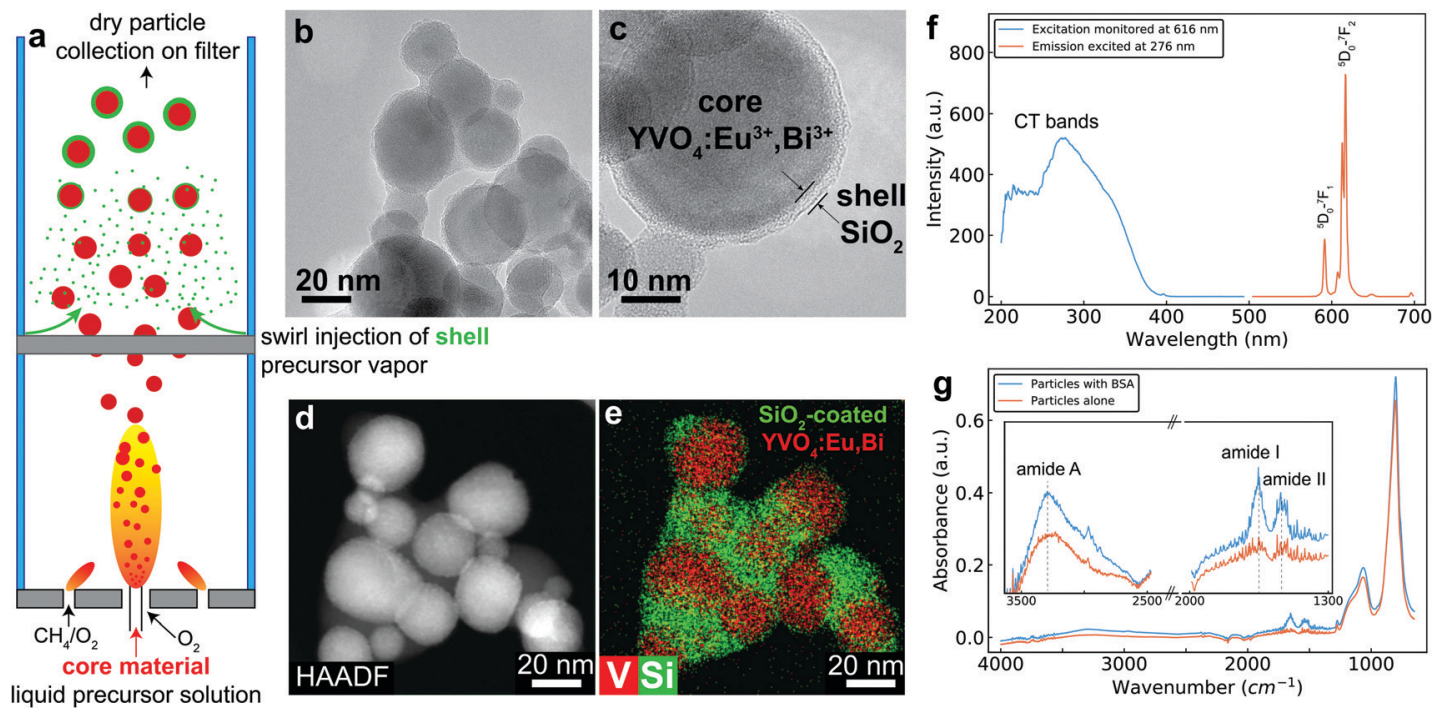

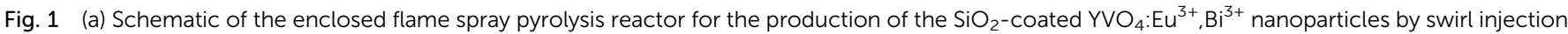
of the Si-precursor vapor downstream after formation of core nanoparticles. (b and c) Transmission electron microscopy images of the $\mathrm{SiO}_{2}$-coated $\mathrm{YVO}_{4}: \mathrm{Eu}^{3+}, \mathrm{Bi}^{3+}$ nanoparticles in which the characteristic amorphous nanothin $\mathrm{SiO}_{2}$ layer encapsulates the core phosphor nanoparticles. (d) High-angle annular dark field from a scanning tunneling electron microscopy image and the corresponding elemental mapping (e) highlighting the $\mathrm{V}$ (core) and $\mathrm{Si}$ (shell) elements. (f) The excitation (blue line, monitoring emission at $\lambda=616 \mathrm{~nm}$ ) and emission (red line, excitation at $\lambda=276 \mathrm{~nm}$ ) spectra of the nanophosphors. (g) FTIR-ATR spectra of nanoprobes before (red line) and after (blue line) their functionalization with bovine serum albumin (BSA). 
surface of the nanoprobes and furthermore an increase in the intensity in the region of the amide A band around $3300 \mathrm{~cm}^{-1}$ (inset in Fig. 1g), even though this band could also be attributed to the presence of water. The biofunctionalization is further validated here by the changes in the hydrodynamic diameter of the nanoprobes after the protein biofunctionalization as well as by the bicinchoninic acid (Pierce BCA) assay (ESI, $\uparrow$ Table S1). The protein biofunctionalization does not affect the excitation and emission profile of the nanoprobes (ESI, $\dagger$ Fig. S4).

The suitability of the developed nanoprobes for dynamic bioimaging is evaluated by challenging human lung epithelial cells (A549) with $S$. pneumoniae bacteria that express the green fluorescent protein GFP. $S$. pneumoniae is a main cause of pneumonia, sepsis and meningitis, and one of the most common causes of deaths worldwide. ${ }^{33,34}$ To cause disease, pneumococci interact with human cell barriers such as the alveolar epithelium in pneumonia ${ }^{35}$ or the blood-brain barrier in meningitis. ${ }^{34}$ Thus, it is important to monitor its interactions with host cells over time. To visualize the cell barrier, host cells have been pre-labelled either with phalloidin that is an organic dye that binds to the cytoskeleton, or with the developed luminescent nanoprobes $(1 \mathrm{~h}$ incubation prior addition of bacteria at $C=30 \mathrm{mg} \mathrm{L}^{-1}$ ). The as-prepared nanoparticles do not exhibit any cytotoxicity for up to $100 \mathrm{mg} \mathrm{L}^{-1}$ (ESI, $\uparrow$ Fig. S5) justifying the imaging dose. Bacteria-host cell interactions are then monitored continuously for $30 \mathrm{~min}$, utilizing constant imaging conditions (exposure time and light intensity) as shown in Fig. 2a. Even though the cytoskeleton staining by the organic dye provides a bright image for time $t=0 \mathrm{~min}$, the signal gradually degrades over time, exhibiting photobleaching, and the cells cannot be detected after $30 \mathrm{~min}$. In contrast, the signal of cells stained with nanoprobes does not degrade during the time studied here highlighting their superior photostability, but also rendering it possible for the first time to visualize in real-time and in situ pathogen-host cell interactions (ESI, $\uparrow$ Fig. S6 for further images). Fig. 2b shows red signal from such images, quantitatively validating that the nanoprobes exhibit superior stability over a commonly used organic dye that loses more than 3 times its intensity over the first 5 min of imaging. The performance of the nanoprobes during in vitro bioimaging is not significantly affected by the primary particle

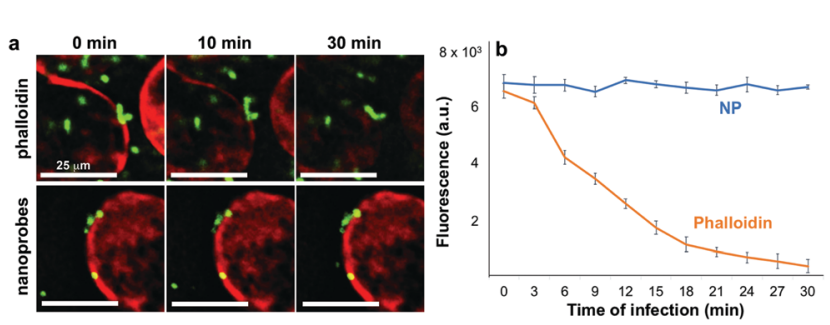

Fig. 2 (a) A549 lung cancer epithelial cells with conventional phalloidin staining (top row, $C=2$ units per $\mathrm{mL}$ ) and with the red nanophosphors (bottom row, $C=30 \mathrm{mg} \mathrm{L}^{-1}$ ), co-incubated with $\mathrm{S}$. pneumoniae expressing GFP (green) over different time points (0, 10, $30 \mathrm{~min})$. Images have same scale bar. (b) Quantitative analysis of the luminescence intensity signal originating from the red fluorescence emission filter for both these cell cultures stained with the nanoprobes (NP) and phalloidin. $N \geq 2$. size uniformity given the rather homogeneous signal from the cell membrane upon its labeling with the nanoprobes.

Furthermore, the functionality of the developed nanoprobes here is evaluated by selectively targeting the cytoskeleton of lung epithelial cells utilizing nanoprobes functionalized with antiCK8 antibody. The cells are incubated for $1 \mathrm{~h}$ at $37{ }^{\circ} \mathrm{C}$ with three formulations $\left(C=50 \mathrm{mg} \mathrm{L}^{-1}\right.$, see ESI, $\dagger$ Fig. $\mathrm{S} 7$ for $100 \mathrm{mg} \mathrm{\textrm {L } ^ { - 1 }}$ ): (i) pure, non-functionalized nanoprobes (NPs only), (ii) antiCK8 functionalized nanoprobes (antiCK8-NPs) and (iii) nanoprobes functionalized with BSA (BSA-NPs) that was used as a negative control due to the high biocompatibility and minimal interaction of albumin with host cells. ${ }^{36}$ Afterwards, the cultures are washed and placed in the fluorescence microscope, and the nanoprobe luminescence is monitored to study their interaction with the host cells. Fig. 3a shows representative transmission-DIC, red luminescence and the merged microscope images from these conditions for two different cell culture orientations: (i) upright and (ii) inverted.

The quantified signal intensity from these images is shown in Fig. 3b. Upon the traditional upright cell culture orientation (the cells grow on the bottom of the wells), there is a strong nanoprobe signal from both the NPs only and the antiCK8-NPs. Pure, non-functionalized nanoprobes readily interact with the cells due to particle sedimentation and other non-specific pathways ${ }^{23,37}$ however, when the nanoprobes are functionalized with the targeting antiCK8 antibody, their cell-associated amount is drastically increased ( $\sim 2$-fold). The nanoprobes seem to be internalized by the cells as signals are detected continuously from the apical to the basolateral side (ESI, $\dagger$ Fig. S8). When the BSA-NPs are employed, there is almost no

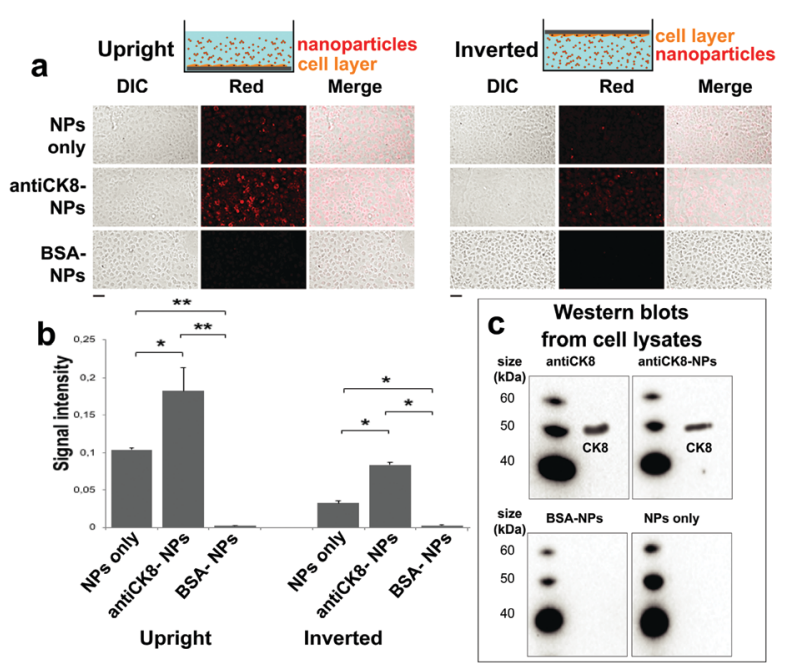

Fig. 3 (a) Microscopy images of A549 cells incubated with $50 \mathrm{mg} \mathrm{L}^{-1}$ of (i) pure nanophosphors, (ii) biofunctionalized with antiCK8, and (iii) biofunctionalized with BSA at upright (cells bottom of well) and inverted cell orientation (cells on top) displaying the DIC (left column), red fluorescence (middle column) and merge images (right column). Scale bar is $25 \mu \mathrm{m}$. (b) Quantitative analysis of the nanoparticle uptake from these images monitoring the red fluorescence, $N=3$. (c) Western blots from A549 cell lysates incubated with (i) pure antiCK8 antibody, (ii) nanoprobes, (iii) nanoprobes biofunctionalized with antiCK8, and (iv) biofunctionalized with BSA. 
signal, indicating that the BSA functionalization on the nanoprobes effectively inhibits any interaction between the nanoprobes and the cells. ${ }^{38}$

To further study the targeting capacity of the antibodyfunctionalized nanoprobes, an inverted cell culture orientation is employed in which the cells grow on top of the wells (see scheme in Fig. 3). This way, any interaction between cells and nanoprobes due to particle sedimentation is avoided and only interactions by Brownian diffusion are taken into account. ${ }^{23,37,39}$ Indeed, the signal intensity of non-functionalized nanoprobes (NPs only) in inverted orientation is $>4$ times decreased when compared to that of upright orientation indicating their reduced interaction with the cells. Most importantly, the interaction of antiCK8-NPs in the inverted cell orientation is further enhanced by almost 3-fold than the uncoated ones indicating the targeting capacity/affinity of the antibody-functionalized nanoprobes towards the cells. Similar to the upright cell orientation, the BSA-NPs exhibit minimal interaction with the cells. Finally, to further validate that the antiCK8 antibody is indeed delivered to the cells when functionalized on the nanoprobes, we measure the western blots of the cell lysates upon incubation of the three different nanoprobe formulations along with the pure antibody. Fig. $3 c$ shows these western blots in which the presence of the antiCK8 antibody is detected only when cells are incubated either with the pure antibody or with the antibodyfunctionalized nanoprobes.

In summary, we develop a novel silica coated nanoprobe suitable for in vitro dynamic bioimaging with conventional fluorescence microscopes. These nanoprobes exhibit superior optical photostability, rendering it possible to monitor pathogen-host cell interactions over time without the loss of any imaging signal. The biocompatible amorphous silica coating facilitates the surface biofunctionalization of these nanoprobes, and the targeting of biomolecules is demonstrated by selectively targeting lung epithelial cells with antiCK8 functionalized nanoprobes in both upright and inverted cell culture orientations. These novel luminescent nanoprobes could help elucidating mechanisms for how bacterial pathogens interact with host cells, and constitute a valuable tool in nanomedicine research.

\section{Conflicts of interest}

There are no conflicts to declare.

\section{References}

1 J. C. G. Bunzli, J. Lumin., 2016, 170, 866-878.

2 M. Montalti, A. Cantelli and G. Battistelli, Chem. Soc. Rev., 2015, 44, 4853-4921.

3 S. Veeranarayanan, A. C. Poulose, S. Mohamed, A. Aravind, Y. Nagaoka, Y. Yoshida, T. Maekawa and D. S. Kumar, J. Fluoresc. 2012, 22, 537-548.

4 G. F. Wang, Q. Peng and Y. D. Li, Acc. Chem. Res., 2011, 44, 322-332.

5 O. S. Wolfbeis, Chem. Soc. Rev., 2015, 44, 4743-4768.
6 A. Escudero, C. Carrillo-Carrion, M. V. Zyuzin and W. J. Parak, Top. Curr. Chem., 2016, 374, 48.

7 X. Y. Liu, Y. Li, C. K. Tsung and J. Li, Chem. Commun., 2019, 55, 10669-10672.

8 G. A. Sotiriou, M. Schneider and S. E. Pratsinis, J. Phys. Chem. C, 2011, 115, 1084-1089.

9 D. F. Henning, P. Merkl, C. H. Yun, F. Iovino, L. Xie, E. Mouzourakis, C. Moularas, Y. Deligiannakis, B. Henriques-Normark, K. Leifer and G. A. Sotiriou, Biosens. Bioelectron., 2019, 132, 286-293.

10 A. Pratsinis, G. A. Kelesidis, S. Zuercher, F. Krumeich, S. Bolisetty, R. Mezzenga, J. C. Leroux and G. A. Sotiriou, ACS Nano, 2017, 11, 12210-12218.

11 G. Blasse, J. Chem. Phys., 1966, 45, 2356-2360.

12 J. Pan, D. Wan, Y. Bian, H. Sun, C. Zhang, F. Jin, Z. Huang and J. Gong, AIChE J., 2013, 59, 4494-4501.

13 G. A. Sotiriou, D. Franco, D. Poulikakos and A. Ferrari, ACS Nano, 2012, 6, 3888-3897.

14 T. Ohno, Y. Iso and T. Isobe, ECS J. Solid State Sci. Technol., 2016, 5, R142-R145.

15 W. J. Park, M. K. Jung and D. H. Yoon, Sens. Actuators, B, 2007, 126, 324-327.

16 A. Escudero, C. Carrillo-Carrion, M. V. Zyuzin, S. Ashraf, R. Hartmann, N. O. Nunez, M. Ocana and W. J. Parak, Nanoscale, 2016, 8, 12221-12236.

17 Y. C. Chen, S. C. Huang, Y. K. Wang, Y. T. Liu, T. K. Wu and T. M. Chen, Chem. - Asian J., 2013, 8, 2652-2659.

18 A. Spyrogianni, P. Tiefenboeck, F. H. L. Starsich, K. Keevend, F. Krumeich, I. K. Herrmann, J. C. Leroux and G. A. Sotiriou, AIChE J., 2018, 64, 2947-2957.

19 K. Keevend, L. Puust, K. Kurvits, L. R. H. Gerken, F. H. L. Starsich, J. H. Li, M. T. Matter, A. Spyrogianni, G. A. Sotiriou, M. Stiefel and I. K. Herrmann, Nano Lett., 2019, 19, 6013-6018.

20 A. Teleki, M. C. Heine, F. Krumeich, M. K. Akhtar and S. E. Pratsinis, Langmuir, 2008, 24, 12553-12558.

21 G. A. Sotiriou, A. M. Hirt, P. Y. Lozach, A. Teleki, F. Krumeich and S. E. Pratsinis, Chem. Mater., 2011, 23, 1985-1992.

22 F. Iovino, D. L. Hammarlof, G. Garriss, S. Brovall, P. Nannapaneni and B. Henriques-Normark, J. Clin. Invest., 2016, 126, 2821-2826.

23 A. Spyrogianni, I. K. Herrmann, M. S. Lucas, J.-C. Leroux and G. A. Sotiriou, Nanomedicine, 2016, 11, 2483-2496.

24 S. E. Pratsinis, AIChE J., 2010, 56, 3028-3035.

25 A. Teleki, M. Suter, P. R. Kidambi, O. Ergeneman, F. Krumeich, B. J. Nelson and S. E. Pratsinis, Chem. Mater., 2009, 21, 2094-2100.

26 G. A. Sotiriou, T. Sannomiya, A. Teleki, F. Krumeich, J. Vörös and S. E. Pratsinis, Adv. Funct. Mater., 2010, 20, 4250-4257.

27 G. A. Sotiriou, F. Starsich, A. Dasargyri, M. C. Wurnig, F. Krumeich, A. Boss, J.-C. Leroux and S. E. Pratsinis, Adv. Funct. Mater., 2014, 24, 2818-2827.

28 G. A. Sotiriou, M. Schneider and S. E. Pratsinis, J. Phys. Chem. C, 2012, 116, 4493-4499.

29 A. Teleki, M. K. Akhtar and S. E. Pratsinis, J. Mater. Chem., 2008, 18, 3547-3555.

30 R. Cagliani, F. Gatto and G. Bardi, Materials, 2019, 12, 1991

31 M. Tonigold, J. Simon, D. Estupinan, M. Kokkinopoulou, J. Reinholz, U. Kintzel, A. Kaltbeitzel, P. Renz, M. P. Domogalla, K. Steinbrink, I. Lieberwirth, D. Crespy, K. Landfester and V. Mailander, Nat. Nanotechnol., 2018, 13, 862-869.

32 J. Grdadolink and Y. Marechal, Biopolymers, 2001, 62, 40-53.

33 B. Henriques-Normark and E. I. Tuomanen, Cold Spring Harbor Perspect. Med., 2013, 3, a010215.

34 F. Iovino, J. Seinen, B. Henriques-Normark and J. M. van Diji, Trends Microbiol., 2016, 24, 307-315.

35 P. Shivshankar, C. Sanchez, L. F. Rose and C. J. Orihuela, Mol. Microbiol., 2009, 73, 663-679.

36 F. F. An and X. H. Zhang, Theranostics, 2017, 7, 3667-3689.

37 A. Spyrogianni, G. A. Sotiriou, D. Brambilla, J.-C. Leroux and S. E. Pratsinis, J. Aerosol Sci., 2017, 108, 56-66.

38 J. Mariam, S. Sivakami and P. M. Dongre, Drug Delivery, 2016, 23 , 2668-2676.

39 N. Feliu, X. Sun, R. A. A. Puebla and W. J. Parak, Langmuir, 2017, 33, 6639-6646. 